\title{
Proportionally adjusted marginal pricing method to share joint costs
}

\author{
YunTong Wang \\ Faculty of Arts and Social Sciences, Sabanci University, Orhanli 81474 Tuzla / Istanbul-Turkey \\ (e-mail: wang@ sabanciuniv.edu)
}

Received: 23 March 2001 / Accepted: 16 November 2001

\begin{abstract}
We consider the cost sharing problem with divisible demands of heterogeneous goods. We propose a cost sharing method called Proportionally Adjusted Marginal Pricing (PAMP) method. PAMP is a nonadditive (in the cost function) extension of average cost pricing. We introduce an axiom called Local Independence (LI) and use LI together with Continuity, Proportionality, and Scale Invariance to characterize PAMP.
\end{abstract}

JEL classification: C71, D62, D63

Key words: Nonadditive cost sharing methods

\section{Introduction}

Consider the cost sharing model with divisible demands of heterogeneous goods. In this model, the Aumann-Shapley pricing method (Tauman 1988) has been shown to be the only extension of average cost pricing within the family of cost sharing methods satisfying Shapley's (1953) two basic axioms, Additivity and Dummy. Recently, within the same family alternative cost sharing methods have been proposed and characterized by selecting other equity and /or incentive axioms (Moulin and Friedman 1999). At the same time, incompatibility results between Additivity and various combinations of other relevant axioms have been found (e.g., Moulin and Friedman 1999). As a structural invariance axiom, Additivity has played a central role in the literature of axiomatic cost sharing but at the same time has ruled

I thank Yves Sprumont, Hervé Moulin, and Ahmet Alkan for discussions, comments and suggestions. Thanks also go to the associate editor and the referees, whose comments and suggestions greatly improve the paper. 
out potentially meaningful nonadditive cost sharing methods (Moulin 1999). There has been a growing interest in searching for nonadditive cost sharing methods. For example, Sprumont (1998) has proposed a few nonadditive methods including a nonadditive extension of average cost pricing in the two-agent case.

This paper proposes and axiomatizes an alternative nonadditive extension of average cost pricing called Proportionally Adjusted Marginal Pricing (PAMP). PAMP simply normalizes the marginal cost pricing into a cost sharing method. More precisely, PAMP is defined as a weighted average cost pricing, where the quantity demanded by each agent is weighted by its marginal cost.

To obtain a characterization of PAMP, we introduce a Local Independence axiom, which replaces the Additivity axiom used in the characterization of the Aumann-Shapley pricing method (e.g., Billera and Heath 1982; Samet and Tauman 1982). Specifically, we characterize PAMP by Local Independence, Continuity, Proportionality, and Scale Invariance. The Continuity is a technical condition but it implies the traditional Dummy axiom.

Local Independence (LI) axiom requires that cost shares be invariant with changes in the cost function that do not affect the value nor the derivatives of the cost function at the given demand profile. Also a structural invariance axiom, LI leaves rooms for nonadditive methods. Interestingly by combining with other axioms (Dummy is replaced by Continuity) used in the characterization of the Aumann-Shapley, LI characterizes PAMP.

\section{The model}

Let $N=\{1, \ldots, n\}$ be the set of agents. Let $R_{+}^{N}$ be the nonnegative orthant of $R^{N}$, and let $R_{++}^{N}$ be the positive orthant of $R^{N}$. A demand vector is a vector $q \in R_{+}^{N}$. Denote $\mathcal{C}_{0}(N)$ the set of functions $C: R_{+}^{N} \rightarrow R_{+}$, nondecreasing with $C(0)=0$. Denote $\partial_{i} C(\cdot)=\partial C / \partial q_{i}(i \in N)$ when $C$ is differentiable and $\nabla C(q)=\left(\partial_{1} C, \ldots, \partial_{n} C\right) .{ }^{1}$ Denote $\mathcal{C}_{1}(N)$ the set of all continuously differentiable functions in $\mathcal{C}_{0}(N)$ with $\nabla C(q) \neq 0, \quad \forall q \in R_{+}^{N}$. Denote $\mathcal{C}_{1}^{+}(N)$ the set of all continuously differentiable functions in $\mathcal{C}_{0}(N)$ with $\nabla C(q) \in R_{++}^{N}, \quad \forall q \in R_{+}^{N}$. A (cost sharing) problem is a list $(N ; q ; C)$ (or simply $(q ; C)$ if $N$ is fixed) where $q \in R_{+}^{N}$ and $C \in C_{0}(N)$. A (cost sharing) method is a mapping $x$ that assigns to each problem $(q ; C)$ a vector $x(q ; C)$ in $R_{+}^{N}$ such that

$$
\sum_{i \in N} x_{i}(q ; C)=C(q) \text {. }
$$

\section{Axioms}

We propose the following axiom.

Local Independence (LI). For any $q \in R_{+}^{N}$, and any $C^{1}, C^{2} \in \mathcal{C}_{1}(N)$, if $C^{1}(q)$ $=C^{2}(q)$ and $\nabla C^{1}(q)=\nabla C^{2}(q)$, then

$$
x\left(q ; C^{1}\right)=x\left(q ; C^{2}\right) .
$$

1 If $q_{i}=0$, it is understood that $\partial_{i} C(q)$ stands for the right-hand derivative. 
Apparently, LI is a structural invariance axiom. It says that cost shares are invariant with any changes in the cost function as long as there are neither changes in the value nor in the derivatives of the cost function at the given demand profile. However, LI does not rule out the possibility that cost shares may be affected by those changes in the cost function that may eventually affect the derivatives of the cost function at the demand profile.

We will combine LI with the following two well-known axioms.

Proportionality. If $C \in C_{0}(N)$ is homogeneous, i.e., there is a mapping $c: R_{+} \rightarrow$ $R_{+}$such that

$$
C(q)=c\left(\sum_{i \in N} q_{i}\right), \quad \forall q \in R_{+}^{N},
$$

then

$$
x_{i}(q ; C)=\frac{q_{i}}{\sum_{j \in N} q_{j}} c\left(\sum_{j \in N} q_{j}\right), i \in N .
$$

Scale Invariance. For any $q \in R_{+}^{N}$, and any $\lambda \in R_{++}^{N}$.

$$
x(q ; C)=x\left(\lambda q ; C^{\lambda}\right)
$$

where $\lambda q=\left(\lambda_{1} q_{1}, \ldots, \lambda_{n} q_{n}\right)$ and

$$
C^{\lambda}(p)=C\left(\lambda_{1}^{-1} p_{1}, \ldots, \lambda_{n}^{-1} p_{n}\right), \quad \forall p \in R_{+}^{N} .
$$

\section{The PAMP and the Aumann-Shapley pricing}

In marginal cost pricing, agents are assigned with the costs determined by: $\partial_{i} C(q) q_{i}$, $i \in N$. The sum of these costs may not always equal to the total cost $C(q)$, and therefore marginal cost pricing is not a cost sharing method. On the other hand, the average cost pricing (1) defined for the homogeneous goods model can not be used directly in our heterogeneous goods model. Now we normalize the marginal cost pricing into a cost sharing method in the following way.

Definition 1. The Proportionally Adjusted Marginal Pricing method (PAMP) is defined by the following formula:

$$
x_{i}^{P}(q ; C)=\frac{\partial_{i} C(q) q_{i}}{\sum_{j \in N} \partial_{j} C(q) q_{j}} C(q), i \in N .
$$

Thus, the PAMP can be regarded as a weighted average cost pricing with each agent's demand weighted by its marginal cost.

A well-known extension of average cost pricing in the literature is the AumannShapley pricing method defined by:

$$
x_{i}^{A S}(q, C)=q_{i} \int_{0}^{1} \partial_{i} C(t q) d t, i \in N .
$$

The Aumann-Shapley pricing method has been characterized by Proportionality, Scale Invariance, Additivity, and Dummy. ${ }^{2}$

2 Additivity: For any $q$ and any $C^{1}, C^{2}, x\left(q ; C^{1}+C^{2}\right)=x\left(q ; C^{1}\right)+x\left(q ; C^{2}\right)$; Dummy: For any $q, C$, and any $i \in N$, if $\partial_{i} C(\cdot)=0$ then $x_{i}(q ; C)=0$. 


\section{The characterization of PAMP}

First, we characterize the PAMP on the domain $\mathcal{C}_{1}^{+}(N)$.

Theorem 1. There exists a unique cost sharing method satisfying Local Independence, Proportionality and Scale Invariance (on the domain $\mathcal{C}_{1}^{+}(N)$ ). It is the Proportionally Adjusted Marginal Pricing method.

Proof. It is easy to check that the method $x^{P}$ defined by (2) satisfies these axioms. Now we show that any method $\tilde{x}$ which satisfies these axioms will coincide with $x^{P}$.

Consider the family of cost functions defined by:

$$
C_{\alpha}(p)=\left(\sum_{i \in N} p_{i}\right)^{\alpha}, p \in R_{+}^{N}, \quad \text { with } \quad \alpha>0
$$

By Proportionality, $\tilde{x}$ coincides with $x^{P}$ on this family. By Scale Invariance, it also coincides with $x^{P}$ for every cost function of the form:

$$
C_{\lambda ; \alpha}(p)=\left(\sum_{i \in N} \lambda_{i} p_{i}\right)^{\alpha}, p \in R_{+}^{N}, \quad \text { with } \quad \alpha>0, \lambda_{i}>0, i \in N \text {. }
$$

Indeed, the problem $\left(q ; C_{\lambda, \alpha}\right)$ is equivalent to $\left(\lambda q ; C_{\alpha}\right)$ in the sense that

$$
C_{\lambda, \alpha}(p)=C_{\alpha}(\lambda p), \quad \forall p \in R_{+}^{N}
$$

and

$$
C_{\lambda, \alpha}(q)=C_{\alpha}(\lambda q)
$$

Thus, by Scale Invariance and Proportionality,

$$
\begin{aligned}
\tilde{x}_{i}\left(q ; C_{\lambda, \alpha}\right) & =\tilde{x}_{i}\left(\lambda q ; C_{\alpha}\right) \\
& =\frac{\lambda_{i} q_{i}}{\sum_{j \in N} \lambda_{j} q_{j}} C_{\alpha}(\lambda q) \\
& =\frac{\partial_{i} C_{\lambda, \alpha}(q) q_{i}}{\sum_{j \in N} \partial_{j} C_{\lambda, \alpha}(q) q_{j}} C_{\lambda, \alpha}(q) \\
& =x_{i}^{P}\left(q ; C_{\lambda, \alpha}\right), \quad \forall i \in N .
\end{aligned}
$$

Given an arbitrary cost function $C$ in $\mathcal{C}_{1}^{+}(N)$ and any $q \in R_{+}^{N}$, we shall show that there exists a unique $(\lambda, \alpha) \in R_{++}^{N} \times R_{++}$such that

$$
C_{\lambda, \alpha}(q)=C(q) \quad \text { and } \quad \nabla C_{\lambda, \alpha}(q)=\nabla C(q) .
$$

Thus, invoking Local Independence, we shall be able to conclude that

$$
\tilde{x}(q ; C)=\tilde{x}\left(q ; C_{\lambda, \alpha}\right)=x^{P}\left(q ; C_{\lambda, \alpha}\right)=x^{P}(q ; C) .
$$


Substituting the definition of $C_{\lambda, \alpha}$ into (4) yields the following systems of equations

$$
\begin{gathered}
\left(\sum_{j \in N} \lambda_{j} q_{j}\right)^{\alpha}=C(q) \\
\alpha\left(\sum_{j \in N} \lambda_{j} q_{j}\right)^{\alpha-1} \lambda_{i}=\partial_{i} C(q), i \in N .
\end{gathered}
$$

Using the first equation of this system, one can rewrite the second set as

$$
\frac{\alpha \lambda_{i}}{\sum_{j \in N} \lambda_{j} q_{j}}=\frac{\partial_{i} C(q)}{C(q)}, i \in N .
$$

Multiplying (5) by $q_{i}$ and summing yields

$$
\alpha=\bar{\alpha}:=\frac{\sum_{i \in N} \partial_{i} C(q) q_{i}}{C(q)}>0 .
$$

Substituting this value of $\alpha$ back into (5) yields the following system of linear equations

$$
\sum_{j \in N} \lambda_{j} q_{j}=\frac{\sum_{j \in N} \partial_{j} C(q) q_{j}}{\partial_{i} C(q)} \lambda_{i}, i \in N .
$$

Clearly, for any $\gamma>0, \gamma\left(\partial_{i} C(q)\right)_{i \in N}$ is a positive solution of this system. For an appropriate value $\bar{\gamma}$ of $\gamma, \bar{\gamma}\left(\partial_{i} C(q)\right)_{i \in N}$ is also a positive solution of

$$
\left(\sum_{j \in N} \lambda_{j} q_{j}\right)^{\bar{\alpha}}=C(q)
$$

The theorem is proved.

Next, we extend our characterization to the larger domain $\mathcal{C}_{1}(N)$ using a technical condition called Continuity: $x$ is continuous with respect to $\nabla C(q)$.

Theorem 2. The Proportionally Adjusted Marginal Pricing method is the unique cost sharing method satisfying Local Independence, Continuity, Proportionality and Scale Invariance (on the domain $\mathcal{C}_{1}(N)$ ).

Proof. The PAMP is uniquely determined by Local Independence, Proportionality and Scale Invariance on the domain $\mathcal{C}_{1}^{+}(N)$. Then by Continuity, it is uniquely extended to the domain $\mathcal{C}_{1}(N)$.

The theorem is proved.

We now consider the tightness of the characterization.

1) Dropping Local Independence

The Aumann-Shapley pricing method obviously satisfies all the remaining axioms. 
2) Dropping Continuity

a) if $C$ is homogeneous, let

$$
x_{i}(q ; C)=\frac{q_{i}}{\sum_{j \in N} q_{j}} C(q), i \in N
$$

b) if $C$ is not homogeneous, then define

$$
x_{i}^{N}(q ; C)=\left\{\begin{array}{l}
\max \left\{\beta_{1}, \partial_{i} C(q) q_{i}\right\} \text { if } C(q) \geq \sum_{j \in N} \partial_{j} C(q) q_{j} \\
\min \left\{\beta_{2}, \partial_{i} C(q) q_{i}\right\} \text { if } C(q)<\sum_{j \in N} \partial_{j} C(q) q_{j}
\end{array}\right.
$$

where $\beta_{1}$ is the solution of equation:

$$
\sum_{j \in N} \max \left\{\beta, \partial_{j} C(q) q_{j}\right\}=C(q),
$$

and $\beta_{2}$ is the solution of equation:

$$
\sum_{j \in N} \min \left\{\beta, \partial_{j} C(q) q_{j}\right\}=C(q),
$$

and let

$$
x_{i}(q ; C)=x_{i}^{N}(q ; C), \quad \forall i \in N .
$$

3) Dropping Proportionality

For any $q$ and any $C$, let $N^{0}=\left\{i \mid \partial_{i} C(\cdot)=0\right\}, N^{\prime}=N \backslash N^{0}$. Define

a) for $i \in N^{0}$,

$$
x_{i}(q ; C)=0 .
$$

b) for $i \in N^{\prime}$,

$$
x_{i}(q ; C)=x_{i}^{N}(q ; C)
$$

where $x_{i}^{N}$ is defined by (6).

4) Dropping Scale Invariance

For any $q$ and any $C$, let $N^{0}=\left\{i \mid \partial_{i} C(\cdot)=0\right\}, N^{\prime}=N \backslash N^{0}$. Define

$$
x_{i}(q ; C)= \begin{cases}\frac{q_{i}}{\sum_{j \in N^{\prime}} q_{j}} C(q) & \text { if } i \in N^{\prime} \\ 0 & \text { if otherwise }\end{cases}
$$

\section{Other properties of PAMP}

It is easy to check that PAMP also satisfies the following properties.

Distributivity axiom (Moulin and Shenker 1999). For any $q \in R_{+}^{N}$ and any $C^{1} \in \mathcal{C}_{1}(N), C^{2} \in \mathcal{C}_{1}(\{1\})$.

$$
x\left(q ; C^{2} \circ C^{1}\right)=x\left(x\left(q ; C^{1}\right) ; C^{2}\right)
$$

where

$$
C^{2} \circ C^{1}(p):=C^{2}\left(C^{1}(p)\right), \quad \forall x \in R_{+}^{N} .
$$


Cost solidarity (Sprumont 1998). Let $C^{1}$ and $C^{2}$ be two cost functions. Suppose there exists a mapping $r: R_{+} \rightarrow R_{+}$such that $C^{2}=r \circ C^{1}$. Then, either

$$
x\left(q ; C^{1}\right) \ll x\left(q ; C^{2}\right),
$$

or

$$
x\left(q ; C^{1}\right)=x\left(q ; C^{2}\right),
$$

or

$$
x\left(q ; C^{1}\right) \gg x\left(q ; C^{2}\right) .
$$

Note that Cost Solidarity suggests a stronger Local Independence condition defined by ${ }^{3}$

$$
\nabla C^{2}(q)=\gamma \nabla C^{1}(q) \quad \text { with } \quad \gamma \neq 0 \Rightarrow \frac{x\left(q ; C^{2}\right)}{C^{2}(q)}=\frac{x\left(q ; C^{1}\right)}{C^{1}(q)} .
$$

This stronger Local Independence condition is satisfied by PAMP and it implies both Cost Solidarity and Local Independence.

Consistency (Thomson 1996). For all $N, N^{\prime}$ with $N^{\prime} \subseteq N$, all $q \in R_{+}^{N}, C \in$ $\mathcal{C}_{1}(N)$. If $x=x(q ; C)$, we have

$$
x^{N^{\prime}}=x^{N^{\prime}}\left(q_{N^{\prime}} ; r_{N^{\prime}}^{x}(C)\right)
$$

where $x^{N^{\prime}}$ is the restriction of $x$ to $N^{\prime}$, and

$$
r_{N^{\prime}}^{x}(C)\left(y_{N^{\prime}}\right):=\max \left\{C\left(y_{N^{\prime}}, q_{N \backslash N^{\prime}}\right)-\sum_{i \in N \backslash N^{\prime}} x_{i}(q ; C), 0\right\}, \quad \forall y_{N^{\prime}} \in R_{+}^{N^{\prime}}
$$

and we assume that $r_{N^{\prime}}^{x}(C)(\cdot)$ is continuously differentiable at $q_{N^{\prime}}$.

\section{References}

Aumann, R. J., Shapley, L. (1974) Values of nonatomic games. Princeton University Press, Princeton Billera, L., Heath, D. (1982) Allocation of shared costs: A set of axioms yielding a unique procedure. Mathematics of Operations Research 7: 32-39

Friedman, E., Moulin, H. (1999) Three additive methods to share joint costs or surplus. Journal of Economic Theory 87: 275-312

Moulin, H., Shenker, S. (1994) Average cost pricing versus serial cost sharing: An axiomatic comparison. Journal of Economic Theory 64: 178-201

Moulin, H., Shenker, S. (1999) Distributive and additive costsharing of an homogeneous good. Games and Economic Behavior 27 (2): 299-330

Moulin, H. (1999) Axiomatic cost and surplus sharing. In: Arrow, K., Sen, A., Suzumura, K. (eds.) Handbook of Social Choice and Welfare (to appear)

Samet, D., Tauman, Y. (1982) The determination of marginal cost prices under a set of axioms. Econometrica 50: 895-909

Shapley, L. S. (1953) A value for $n$-person games. In: Kuhn, H. W., Tucker, A. W. (eds.) Contributions to the theory of games II. Annals of Mathematics Studies 28: 307-317

Sprumont, Y. (1998) Ordinal cost sharing. Journal of Economic Theory 81: 126-162

Tauman, Y. (1988) The Aumann-Shapley prices: A survey. In: Roth, A. (ed.) The Shapley value. Cambridge University Press, Cambridge

Thomson, W. (1996) Consistent allocation rules. Mimeo, University of Rochester

Young, H. P. (1985) Producer incentives in cost allocation. Econometrica 53: 757-765

3 This observation was pointed out to the author from a referee. 
Reproduced with permission of the copyright owner. Further reproduction prohibited without permission. 